\title{
Feasibility of remote magnetic navigation for epicardial ablation
}

\author{
P. Abraham • L. D. Abkenari • E. C. H. Peters • \\ T. Szili-Torok
}

Published online: 31 May 2013

(C) The Author(s) 2013. This article is published with open access at Springerlink.com

\begin{abstract}
Percutaneous epicardial mapping and ablation is an emerging method to treat ventricular tachycardias (VT), premature ventricular complexes (PVC), and accessory pathways. The use of a remote magnetic navigation system (MNS) could enhance precision and maintain safety. This multiple case history demonstrates the feasibility and safety of the MNS-guided epicardial approach in mapping and ablation of ischaemic VT, outflow tract PVCs, and a left-sided accessory pathway. All patients had previously undergone endocardial mapping for the same arrhythmia. MNS could present an advantage from more precise navigation for mapping and maintaining catheter stability during energy application.
\end{abstract}

Keywords Remote magnetic navigation · Epicardial mapping $\cdot$ Radiofrequency ablation

\section{Introduction}

Navigation with the aid of a magnetic navigation system (MNS) is a well-established electrophysiology (EP) method to map and ablate a wide range of cardiac arrhythmias [1,2]. The theoretical advantages of MNS over conventional manual ablation methods are its superior flexibility, steering accuracy, and stability during radiofrequency (RF) energy application in areas where catheter access can be extremely difficult [3-5]. Exposure to fluoroscopy with MNS is significantly lower for both the electrophysiologist and the patient [6]. This contributes to the safety of MNS regarding mechanical catheter complications and risk of radiation. Percutaneous epicardial mapping and ablation is an emerging approach for treating arrhythmias originating

P. Abraham • L. D. Abkenari • E. C. H. Peters · T. Szili-Torok $(\triangle)$ Department of Cardiology, Thoraxcenter, Erasmus Medical Center, 's Gravendijkwal 230, Ba 577,

3015 CE Rotterdam, the Netherlands

e-mail: t.szilitorok@erasmusmc.nl close to the epicardial surface of the heart [7-9]. Some of these arrhythmias can be localised to the epicardium with a novel experimental non-invasive body surface ECG mapping system [10], which would give the possibility to choose the effective epicardial approach first in selected arrhythmias, thus one could spare the time and resources of an unnecessary endocardial ablation attempt.

The aim of this paper is to demonstrate the MNS-guided epicardial approach and to show the feasibility of this combined method with the following case series. Our three cases involve ablation of an ischaemic ventricular tachycardia (VT), premature ventricular complexes (PVCs) originating from the left ventricular outflow tract (LVOT), and a left-sided accessory pathway. All patients had undergone an endocardial study, two had been ablated.

\section{Methods}

Preprocedural arrangements

All patients were under general anaesthesia and a cardiothoracic surgical team was on standby during the procedure. Patients were informed of the additional risks of an epicardial approach. Coronary angiograms were performed prior to energy application.

Patient preparation

Both groins were prepared for percutaneous punctures. $6 \mathrm{Fr}$ sheaths were used to gain right femoral venous access for standard diagnostic EP catheters, used for diagnostics and as a reference for the electroanatomical mapping system. An $8.5 \mathrm{Fr}$ arterial sheath was inserted in the femoral artery. The chest and abdomen around the xyphoid process were prepared and isolated. This preparation could allow surgical access (mini-thoracotomy) if the percutaneous access failed. 
Pericardial puncture

For percutaneous pericardial access a Tuohy needle (18 G, length: $15 \mathrm{~cm}$ ) and a $9 \mathrm{Fr}, 24 \mathrm{~cm}$ Arrow flex sheath were used. Pericardial puncture with the Tuohy needle was guided by fluoroscopy: X-ray $\mathrm{C}$ arm at $90^{\circ}$ latero-lateral projection, and injection of small quantities of contrast during the puncture. A guide wire (Cordis Exchange wire 35") was introduced via the needle. If establishment of a percutaneous access failed after three consecutive attempts, the subxyphoid isolation site served as an incision point for surgical pericardiotomy. After successful access, a soft flexible sheath was inserted to prevent cardiac laceration and the RMT ablation catheter was introduced into the epicardial space. The baseline flow of the irrigation-tip catheter was $1 \mathrm{ml} / \mathrm{min}$, and the fluid from the pericardium was regularly removed.

\section{Catheter positioning}

Intracardiac diagnostic catheters were positioned first as reference catheters into the right ventricular apex (RVA) during VT and ventricular extrasystole (VES) ablation or into the RVA, coronary sinus (CS) and His position during Wolff-Parkinson-White syndrome (WPW) ablation. An open irrigation $3.5-\mathrm{mm}$ tip magnetic ablation catheter (Navistar RMT ThermoCool, Biosense-Webster, Diamond Bar, CA, USA) was advanced into the pericardial space for epicardial mapping purposes. Details of the MNS technique for endocardial ablation of ventricular arrhythmias have been previously described by our group [2, 3]. Non-irrigated catheters can also be used but this could significantly limit lesion formation.

\section{Coronary angiography}

After an arrhythmia substrate was identified as epicardial, a selective coronary angiography (CAG) was performed. For safety reasons, selective angiography was repeated after every RF application. Cannulation of the particular coronary artery supplying the region of interest was usually enough for this procedure.

\section{Epicardial mapping}

Patients were treated with the remote MNS (Niobe, Stereotaxis, StLouis, MO, USA). A shell of the pericardial space was created by using the fast anatomical mapping (FAM) feature of the CARTO 3 RMT system (Biosense-Webster, Diamond Bar, CA, USA). For ischaemic VT the epicardial surface of the left ventricle was densely mapped.
Post -procedure care after epicardial access

Via the existing percutaneous sheath a temporary pericardial drain (PeriVac pericardial tray) was introduced and removed after $8-12 \mathrm{~h}$ if no significant bleeding or fluid drainage occurred. Intrapericardial steroids were not administered routinely. Following an uncomplicated procedure patients were discharged on the second postoperative day.

\section{Case presentations}

Case 1

A 78-year-old male with chronic ischaemic heart disease was referred for recurrent episodes of VT treated by implantable cardioverter defibrillator (ICD) discharges. He had previously undergone an endocardial VT ablation. His recent $\mathrm{CAG}$ showed a chronic total right coronary artery occlusion with good collateral flow and no progression over time. Under treatment with bisoprolol, amiodarone, phenytoin, and lidocaine the clinical VT with a cycle length of $430 \mathrm{~ms}$ was reproducibly induced during the invasive EP study. Endocardial and epicardial mapping indentified a substrate in the inferior scar of myocardial infarction.

Thirty-three RF pulses were applied with a mean energy of $34 \mathrm{~W}$ from both epicardial and endocardial access in order to obtain scar homogenisation. At the conclusion of the procedure the VT was no longer inducible. There was no significant pericardial bleeding during and after the procedure. The patient was discharged home on oral bisoprolol, amiodarone, and phenytoin. At 3-month follow-up the patient was free of sustained ventricular arrhythmias.

\section{Case 2}

A 50-year-old male presented with frequent PVCs causing marked exercise intolerance. His Holter recording revealed a high number of monomorphic PVCs $(27,000 / 24 \mathrm{~h})$ with bigeminy. Left ventricular ejection fraction was normal. Neither syncope nor sustained ventricular arrhythmias were documented, and the family history was unremarkable. During the previous invasive EP study an ablation had not been performed due to the absence of an adequate pace-map. Using the MNS to guide the catheter, a perfect 12 out of 12 pace-map was gained from the anterior epicardial aspect of the LVOT (Fig. 1).

Delivery of three RF applications with an average energy of $27 \mathrm{~W}$ terminated the arrhythmia and rendered it noninducible (also with isoproterenol infusion). The successful ablation site was $7 \mathrm{~mm}$ away from the left main coronary stem (Fig. 1). No acute complications were observed, but in 

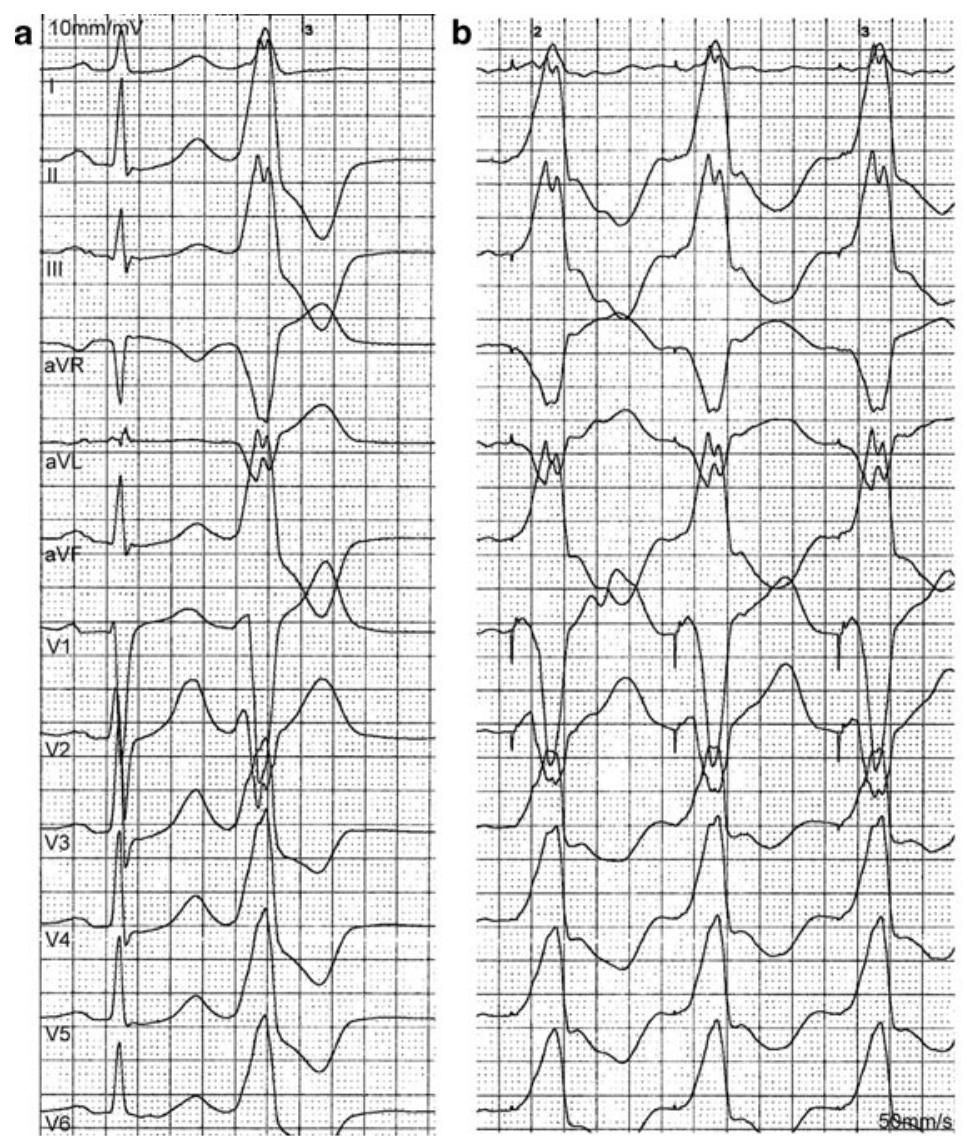

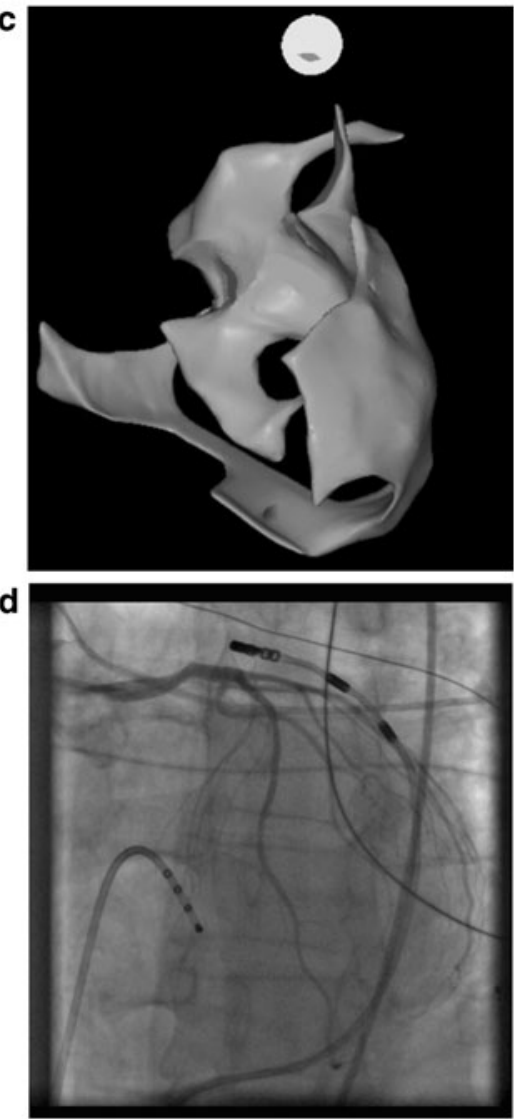

Fig. 1 Ablation of PVCs originating from the LVOT close to the left main stem. Identical ECG pace-map with the spontaneous PVC gained from the successful ablation site (panels a, b). Merged anatomical reconstruction of the left ventricle (inner contour) and the pericardial space (outer contour) created by the FAM module of the electroanatomical mapping system (panel c). The epicardial RF

the early post-procedure period the patient developed a selflimiting sterile pericarditis with an insignificant amount of pericardial fluid. At follow-up after 3 months, PVCs were absent and the patient reported an improved physical condition. His prior antiarrhythmic medication was discontinued.

\section{Case 3}

A 29-year-old male with frequent palpitations underwent three unsuccessful RF ablation procedures of a left-sided accessory pathway. Mapping ablation catheters were introduced epicardially and retrogradely in the left ventricle. After 39 unsuccessful ablation attempts with a mean energy of $27 \mathrm{~W}$, thorough epicardial mapping revealed the earliest atrial retrograde activation site in the left lateral aspect of the mitral annulus.

A single RF application at the corresponding endocardial site by the retrograde catheter abolished the conduction over the accessory pathway. The post-procedural period was uneventful and he was discharged without antiarrhythmic ablation catheter is just $7 \mathrm{~mm}$ away from the main stem of the left coronary artery on the angiogram. A quadripolar electrophysiological reference catheter is in the apex of the right ventricle (panel d). Abbreviations: $P V C$ premature ventricular complex, LVOT left ventricular outflow tract, FAM fast anatomical mapping, $R F$ radiofrequency

medication. In his 3-month follow-up he was completely free of arrhythmias.

\section{Discussion}

To understand the recent developments in MNS one should consider the basic principles of ablation robots and robotics. Introduction of robots in catheter ablation only makes sense if these machines address the most important challenges in clinical cardiac electrophysiology. These challenges include inefficient workflow, inter-operator skill differences and safety issues. The robotic system currently offers a dramatic reduction in fluoroscopy exposure and virtually zero chance of cardiac perforation [11-13]. A significant improvement in robotic manipulation offered by the new Epoch solution, which is commercially available, allows remote manipulation of diagnostic catheters and sheaths [14]. Another development which should take place soon aims at better control of RF energy delivery. In an ideal situation a contact force sensor will 
be included in the next generation of magnetic catheters to make ablation lesions more durable. Navigation in the pericardial space with the aid of MNS is a recent approach which aims to combine the advantages of the two methods in order to increase treatment efficacy and to keep procedurerelated risks and fluoroscopy exposure minimal. Reports on the use of MNS in epicardial mapping and ablation are limited $[15,16]$. Aryana and colleagues used a non-irrigated 4-mm tip magnet-enabled ablation catheter for VT ablation in 20052006. Technical development over the past years has enabled operators to use an open-irrigated magnetic catheter [16].

Indications for an epicardial approach

The setup of percutaneous epicardial catheter ablation can be complex. As a consequence the epicardial technique is commonly used after a failed endocardial attempt. Epicardial approach as a first-line treatment of VT has been increasingly used (35.8\%) according to the results of a recent multicentre European study [17]. Although the likelihood of an epicardial substrate is highest in dilated cardiomyopathy, arrhythmogenic right ventricular cardiomyopathy, and Chagastic myocardial disease, ischaemic cardiomyopathy constitutes the most frequent underlying heart disease $(39 \%)$ which requires epicardial ablation [17]. A VT originating from an inferior infarction scar could potentially be ablated via the percutaneous epicardial approach. Sosa et al. report that post-infarction pericardial adhesions did not limit the movement of the manual mapping catheter [18]. The advantages of MNS are apparent in more precise navigation to difficult-to-reach areas and in maintaining catheter stability during RF applications regardless of the approach.

In our patient with an accessory pathway, the successful ablation was performed from the endocardial side, and only mapping on the epicardial surface revealed the exact site of the left lateral accessory pathway, as retrograde conduction was shown to be altered due to a mitral isthmus block created by a previous ablation within the coronary sinus.

\section{Fluoroscopy exposure with and without MNS}

Total fluoroscopy time was 29 and $27 \mathrm{~min}$ in our VT and PVC cases, respectively. The total procedural fluoroscopy time using MNS was $21 \pm 7 \mathrm{~min}$ according to the single-centre experience of Aryana et al. [15], which was substantially shorter than the mean fluoroscopy time required ( $45 \pm 24 \mathrm{~min}$ ) in another single-centre study with the manual approach [7], or in a multicentre European study $(40 \pm 21 \mathrm{~min})$ where only a mere $4 \%$ of the 218 patients were treated with MNS [17].

Fluoroscopy exposure $(82 \mathrm{~min})$ was substantial to the WPW patient, because electroanatomical mapping was not performed and endocardial mapping of the accessory pathway was misleading.
Another method of reducing procedural fluoroscopy time regardless of the use of MNS is to merge preacquired coronary computed tomography angiograms (CCTA) with the electroanatomical map to avoid multiple CAG expositions as it has been described by Zeppenfeld et al. [19]. Three-dimensional computed tomography image overlay with fluoroscopy is a novel method to create the cardiac anatomy instead of using electroanatomical mapping [20]. While those methods can undoubtedly reduce procedural X-ray exposition, one must take into account the additional radiation exposure during computed tomography scanning.

\section{Conclusion}

Although a considerably higher number of cases are required to undoubtedly demonstrate the efficacy and safety of MNS in epicardial ablation, we believe that the use of MNS has those unique features that could demonstrate its feasibility in difficult epicardial ablation cases.

\section{Funding None.}

\section{Conflict of interests None declared.}

Open Access This article is distributed under the terms of the Creative Commons Attribution License which permits any use, distribution, and reproduction in any medium, provided the original author(s) and the source are credited.

\section{References}

1. Schmidt B, Chun KR, Tilz RR, et al. Remote navigation systems in electrophysiology. Europace. 2008;10 Suppl 3:iii57-61.

2. Anné W, Schwagten B, Janse P, et al. Flutter ablation with remote magnetic navigation: comparison between the $8-\mathrm{mm}$ tip, the irrigated tip and a manual approach. Acta Cardiol. 2011;66:287-92.

3. Thornton AS, Res J, Mekel JM, et al. Use of advanced mapping and remote magnetic navigation to ablate left ventricular fascicular tachycardia. Pac Clin Electrophysiol. 2006;29:685-8.

4. Thornton AS, Jordaens LJ. Remote magnetic navigation for mapping and ablating right ventricular outflow tract tachycardia. Heart Rhythm. 2006;3:691-6.

5. Schwagten B, Szili-Torok T, Rivero-Ayerza M, et al. Usefulness of remote magnetic navigation for ablation of ventricular arrhythmias originating from outflow regions. Neth Heart J. 2009;17:245-9.

6. Arya A, Zaker-Shahrak R, Sommer P, et al. Catheter ablation of atrial fibrillation using remote magnetic catheter navigation: a case-control study. Europace. 2011;13:45-50.

7. Grimard C, Lacotte J, Hidden-Lucet F, et al. Percutaneous epicardial radiofrequency ablation of ventricular arrhythmias after failure of endocardial approach: a 9-year experience. J Cardiovasc Electrophysiol. 2010;21:56-61.

8. Yamada T, McElderry HT, Doppalapudi H, et al. Idiopathic premature ventricular contractions successfully ablated from the epicardial right ventricular outflow tract. Europace. 2011;13:595-7. 
9. Valderrábano M, Cesario DA, Ji S, et al. Percutaneous epicardial mapping during ablation of difficult accessory pathways as an alternative to cardiac surgery. Heart Rhythm. 2004;1:311-6.

10. Oostendorp TF, van Dessel PF, Coronel R, et al. Noninvasive detection of epicardial and endocardial activity of the heart. Neth Heart J. 2011;19:488-91.

11. Szili-Torok T, Akca F. Remote magnetic navigation in atrial fibrillation. Expert Rev Med Devices. 2012;9:249-55.

12. Szili-Torok T, Schwagten B, Akca F, et al. Catheter ablation of ventricular tachycardias using remote magnetic navigation: a consecutive case-control study. J Cardiovasc Electrophysiol. 2012;23:948-54.

13. Bauernfeind T, Akca F, Schwagten B, et al. The magnetic navigation system allows safety and high efficacy for ablation of arrhythmias. Europace. 2011;13:1015-21.

14. Nölker G, Gutleben KJ, Muntean B, et al. Novel robotic catheter manipulation system integrated with remote magnetic navigation for fully remote ablation of atrial tachyarrhythmias: a two-centre evaluation. Europace. 2012;14:1715-8.

15. Aryana A, d'Avila A, Heist EK, et al. Remote magnetic navigation to guide endocardial and epicardial catheter mapping of scarrelated ventricular tachycardia. Circulation. 2007;115:1191-200.
16. Di Biase L, Santangeli P, Astudillo V, et al. Endo-epicardial ablation of ventricular arrhythmias in the left ventricle with the Remote Magnetic Navigation System and the 3.5-mm open irrigated magnetic catheter: results from a large single-center case-control series. Heart Rhythm. 2010;7:1029-35.

17. Della Bella P, Brugada J, Zeppenfeld K, et al. Epicardial ablation for ventricular tachycardia: a European multicenter study. Circ Arrhythm Electrophysiol. 2011;4:653-9.

18. Sosa E, Scanavacca M, d'Avila A, et al. Nonsurgical transthoracic epicardial catheter ablation to treat recurrent ventricular tachycardia occurring late after myocardial infarction. J Am Coll Cardiol. 2000;35:1442-9.

19. Zeppenfeld K, Tops LF, Bax JJ, et al. Epicardial radiofrequency catheter ablation of ventricular tachycardia in the vicinity of coronary arteries is facilitated by fusion of 3-dimensional electroanatomical mapping with multislice computed tomography. Circulation. 2006;114:e51-2.

20. van der Voort PH, Stevenhagen J, Dekker LR, et al. Threedimensional computed tomography overlay for pulmonary vein antrum isolation: Follow-up and clinical outcomes. Neth Heart J. 2012;20:302-6. 